\title{
Stereotactic Ablative Radiotherapy (SABR/SBRT) for Hepatocellular Carcinoma
}

\author{
Byung-Han Rhieu ${ }^{1} \cdot$ Amol K. Narang ${ }^{1} \cdot$ Jeffrey Meyer ${ }^{1}$
}

Published online: 16 October 2018

(C) The Author(s) 2019, corrected publication September 2019

\begin{abstract}
Purpose of Review Liver-directed SABR (stereotactic ablative body radiotherapy) is emerging as an effective local therapy option for HCC (hepatocellular carcinoma). This review summarizes recent clinical progresses and proposes future directions. Recent Findings SABR is an effective and safe, non-invasive local therapy option for HCC in the primary and salvage treatment settings, as well as a bridge to liver transplantation in selected patients. Randomized trials comparing SABR with other locoregional modalities are currently ongoing.

Summary Research efforts are being made toward better predicting normal tissue toxicity and tumor radiosensitivity for a tailored maximal safe treatment in HCC SABR. More recently, potential synergy with immunotherapies is of increasing interest in HCC.
\end{abstract}

Keywords Hepatocellular carcinoma $\cdot \mathrm{HCC} \cdot$ Liver SABR $\cdot$ Liver SBRT $\cdot$ Stereotactic ablative radiotherapy $\cdot$ Stereotactic body radiotherapy

\section{Introduction}

Hepatocellular carcinoma (HCC) is the second leading cause of cancer death worldwide [1]. The prognosis for HCC is poor, with overall ratio of mortality to incidence of 0.95 [1]. Despite its continual rise in incidence, there is still an unmet need for effective therapy. This has been largely due to the advanced stage at diagnosis, aggressive tumor biology, limited systemic therapy options, limitations of existing local therapies, and scarce donor organ availability for those eligible for transplant.

In the USA alone, more than 100,000 individuals are diagnosed with primary liver cancer each year, but less than 20 $30 \%$ are candidates for curative resection due to either technical and or medical inoperability that is often complicated by underlying cirrhosis $[2,3]$. Orthotopic liver transplantation has existed as a durable curative option with a 5-year survival rate exceeding $70 \%$ and recurrence rates of $15 \%$ [4], but is

This article is part of the Topical Collection on Hepatic Cancer

Byung-Han Rhieu

brhieu1@jhmi.edu

1 Department of Radiation Oncology, Johns Hopkins Hospital, 401 North Broadway / Suite 1440, Baltimore, MD 15213, USA limited by donor availability and patient ineligibility. For the past decade, the multikinase inhibitor sorafenib has been the main systemic agents, providing a 2-3-month overall survival benefit in advanced HCC $[5,6]$. Several non-operative locoregional therapy options exist, including radiofrequency ablation (RFA) and microwave ablation, cryoablation, transarterial embolization (TAE) (i.e., bland embolization), transarterial chemoembolization (TACE), radioembolization (Yttrium-90) and percutaneous ethanol injection (PEI). Most recently, a new external beam radiotherapy approach called stereotactic ablative body radiotherapy (SABR), (synonymous with stereotactic body radiation therapy or SBRT) has emerged as a promising modality.

This review highlights recent technological and clinical progress in liver-directed SABR for HCC, discusses ongoing controversies, and proposes future directions drawing from recent development in several domains including immunotherapy and imaging.

\section{Thermal Ablation and Embolization Therapy for HCC}

For unresectable HCC, RFA and TAE/TACE are two commonly used local-regional therapies, with a wide variation in reported survival and local control rates. These therapies are 
discussed in detail elsewhere [7, 8]. Long-term studies of RFA for selected patients show 5-year overall survival of over $50 \%$, and local control rate ranging 59-97\% for tumor size $<5 \mathrm{~cm}$ [7, 9-13]. The utility of RFA is limited by large $(>3 \mathrm{~cm})$ tumor size and the heat sink effect when large blood vessels are near the tumor $[8,10,11,13,14]$. Rare toxicities associated with RFA include abscess formation and injury to surrounding tissues as a function of tumor location.

Embolization therapy takes advantage of the arterial-based perfusion of HCC and has shown survival advantage over supportive care for unresectable HCC in two landmark randomized trials and can induce substantial necrosis in HCC tumors [15, 16]. However, durable long-term tumor control for larger tumors is limited by revascularization within the tumor [8]. Toxicities associated with embolization include the post-embolization syndrome consisting of fever, nausea, abdominal pain, and ileus [17-19]. Sometimes, prolonged hospitalization is required to monitor the patients and to control pain [20].

\section{Advancements in Radiotherapy and a Newly Established Role in HCC Treatment}

Historically, conventionally fractionated external beam radiotherapy was limited to a largely palliative role in $\mathrm{HCC}$, with low local control and overall survival rates. Attempts at improving local control with dose escalation were limited by the risk of radiation-induced liver disease (RILD), a potentially fatal syndrome of hepatomegaly and ascites $[21,22]$. RILD is histopathologically characterized by veno-occlusive disease [23] and is seen more commonly with large-volume liver irradiation.

However, in the past decades, advancements in treatment planning, motion management, and image guidance have allowed for high precision focal irradiation with escalated radiation doses and low risk of toxicity. This success was founded in the principles of intracranial radiosurgery: potent/ ablative radiation treatment delivered in one or a few treatments (hypofractionated, 5 or fewer treatments) with high precision and steep gradient of dose in the surrounding normal tissues [24]. Extracranial stereotactic radiotherapy is now commonly used in the clinic to treat a variety of cancers, including liver tumors. Results from various series of SABR for HCC are summarized in Table 1 [25-29, 30•].

The results from these series show SABR to be an effective treatment for HCC, with reasonable toxicity profiles. Andolino et al. reported on the use of SABR for 60 patients with HCC. Patients with CTP B cirrhosis were treated with more modest hypofractionation. Local control at 2 years using RECIST criteria was $90 \%$. Twenty percent of patients developed progression of their CTP class within 3 months of treatment. Bujold and colleagues used a normal tissue complication probability approach to tailor prescription radiation doses, and showed high rates of local control with minimal toxicities. Takeda and colleagues reported on patients with HCC treated with SABR with or without preceding TACE. Local control at 3 years was high, and less than $10 \%$ of patients had an increase in their CTP score of 2 points.

Classic RILD is rarely seen with modern approaches to liver irradiation. However, "non-classic" RILD, a term largely encompassing any sign of liver injury or functional deterioration, can be observed [31]. Generally, measures of cirrhosis such as the CTP score can be used to select patients for different dose-fractionation treatment regimens, with more aggressive treatments for the patients with well-compensated cirrhosis. Cardenes and colleagues showed poor tolerance to SABR for patients with decompensated cirrhosis, and much of the published literature selects for patients with CTP A or CTP $\mathrm{B}$ cirrhotic states [25]. The primary consideration is sparing of non-tumor involved liver tissue [31, 32]. Integration of patient-specific functional imaging may further aid in the treatment planning process to limit radiation-induced toxicities [33-37]. Selection of dose-fractionation scheme for a given patient remains an active area of investigation.

\section{Comparison with Other Local Therapies}

To date, randomized clinical data directly comparing SABR with RFA for HCC is lacking. Investigators have reported on institutional results and analyzed the National Cancer Database in efforts to address this comparison.

A recent institutional series from the University of Michigan group compared outcomes of RFA and SABR for HCC. In this study, 224 inoperable, non-metastatic HCC patients who underwent RFA or SABR were included. One- and 2-year local control rate for tumors treated with RFA were $83.6 \%$ and $80.2 \%$ vs $97.4 \%$ and $83.8 \%$ for SABR [38•]. On multivariate analysis, treatment with RFA was associated with decreased local control (HR 3.84; $p=0.002$ ). Importantly, tumor size influenced the difference in outcomes between treatment modalities. Among patients with tumors $<2 \mathrm{~cm}$ in size, no significant difference in local control was noted between SBRT and RFA, while among patients with tumor size $\geq 2 \mathrm{~cm}$, RFA was associated with significantly inferior freedom from local progression (HR 3.35; $p=0.025$ ). Results from these studies conflict with a report analyzing outcomes for SABR versus RFA from the National Cancer Database, in which patients treated with RFA had superior 5-year overall survival [39]. The authors used propensity matching to account for known confounders differing between the two groups. However, a multitude of patient-specific features are competing factors for survival in patients with HCC, and there are limitations in accounting for these variables and their impact on the outcome of survival in analysis of large sets of data such as this [40]. 


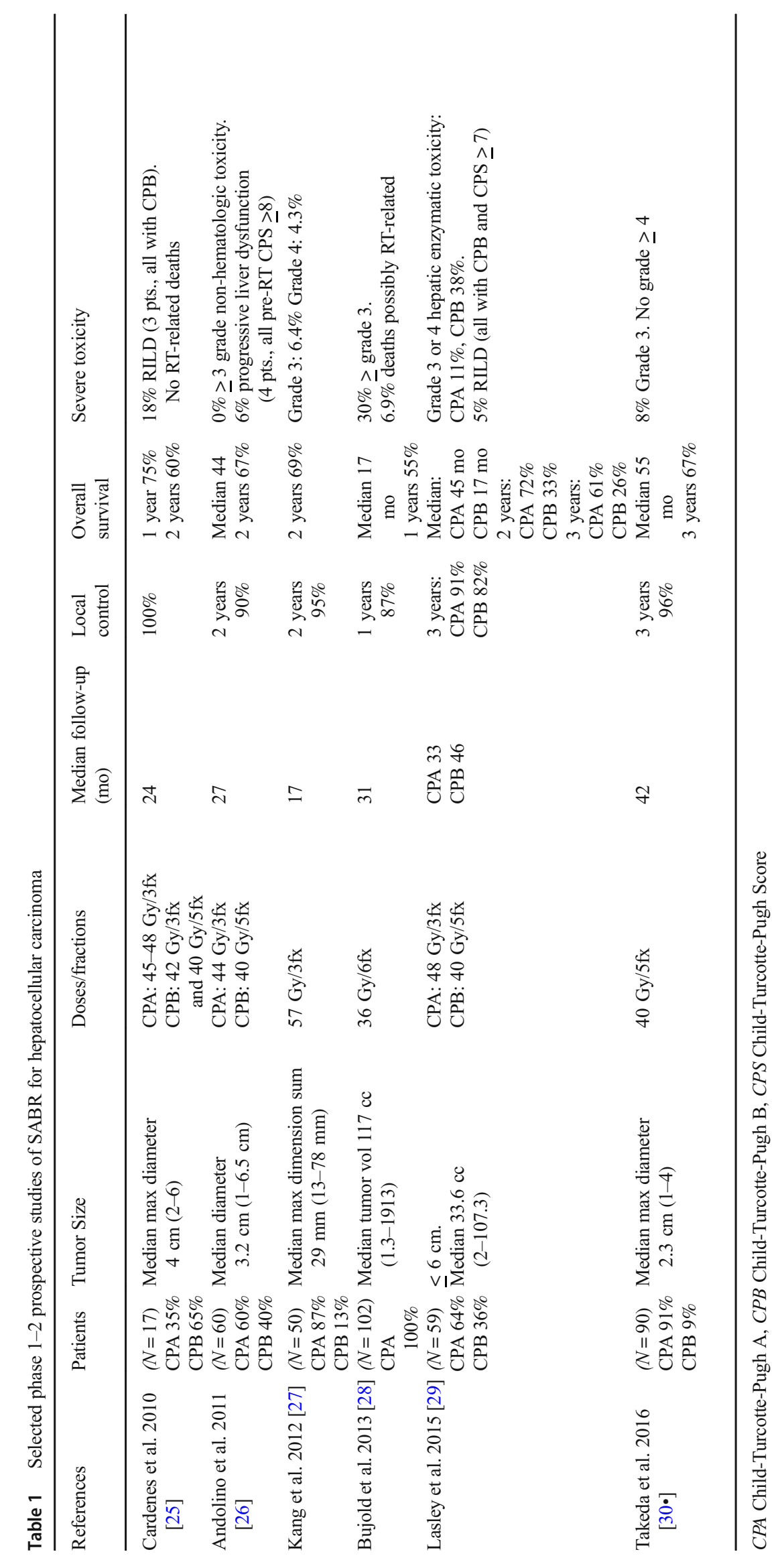


Similarly, Sapir et al. analyzed 209 patients who underwent either SABR or TACE for one to two tumors $(2.3-2.9 \mathrm{~cm})$ [41]. One- and 2-year LC favored SABR: $97 \%$ and $91 \%$, respectively, for SABR and $47 \%$ and $23 \%$ for TACE (hazard ratio $66.5, P<.001$ ), with higher grade $3+$ toxicity with TACE $(13 \%)$ vs SABR $(8 \%)$.

\section{SABR as a Bridging Therapy for Transplant Patients}

The best bridging modality for orthotopic liver transplant is unclear. TACE is most commonly used $[42,43]$. Several recently published works demonstrated SABR as an effective and safe option in selected patients as well [44-49]. Investigators from the Lahey Clinic presented preliminary results of a randomized phase 2 comparison of SABR and TACE prior to OLT at the American Society for Clinical Oncology Gastrointestinal Cancers Symposium (ASCO GI) meeting in 2016 [50•]. At the time of this interim report, 13 patients were treated with SABR and 16 with TACE. Retreatment rates were higher for the patients in the TACE arm, and toxicity and quality of life metrics including elimination of hospitalizations (required for TACE) appeared improved in the SABR arm.

\section{Recognition of SABR as an Alternative for Treatment of Intermediate Stage HCC}

In light of the literature evidence in favor of SABR thus far, the seventh Asia-Pacific Primary Liver Cancer Expert Meeting consensus statement recommended SABR as a safe and effective option [51]. In the USA, the recent NCCN (National Comprehensive Cancer Network) guideline included SABR as an alternative to ablation/embolization for unresectable or medically inoperable HCC [52].

\section{Moderate Hypofractionation}

A hallmark of SABR is the use of high-dose-per-fraction radiation (typically $>8 \mathrm{~Gy} /$ fraction) delivered in a short course (5 or fewer fractions). More moderately hypofractionated regimens (doses around 4 Gy per fraction, delivered over 15 or so fractions), planned delivered with the same guiding principles of SABR, can allow for delivery of very potent treatment courses when the beneficial effects of fractionation are most relevant, such as tumors abutting critical organs. Hong et al. reported on a phase II trial using proton therapy to treat HCC and intrahepatic cholangiocarcinomas in 15 fractions, with proton therapy [53]. Dose-per-fraction was 3.87 Gy or 4.5 Gy depending on tumor location. The median maximum tumor dimension was $5 \mathrm{~cm}$ for the $\mathrm{HCC}$ patients, and local control at 2 years was $94.8 \%$.

Investigators from Loma Linda University reported interim results of a randomized trial comparing 15-fraction proton therapy (4.68 Gy per fraction) with TACE for patients with a new diagnosis of HCC [54]. Two-year progression-free survival was higher in the patients treated with proton therapy ( $48 \%$ versus $31 \%, p=0.06$ ). Patients treated with proton therapy also had fewer hospitalization days compared to patients treated with TACE.

\section{The Problem of Metachronous Disease}

In HCC management, a major challenge is development of disease at other sites in the liver. Even with partial hepatectomy for small tumors, liver recurrence rate exceeds $70 \%$ at 5 years [55]. Explanted liver specimens from patients who undergo OLT following successful local therapy with SABR show high rate of micrometastatic disease in untreated liver not detected on imaging $[44,45]$. Indeed, progression free survival rate of $<50 \%$ is typically observed even when local control of $>85 \%$ is achieved with SABR. These results emphasize the need for better systemic therapies, the latter limited by the dysfunctional cirrhotic liver. However, immunotherapy may change this landscape.

\section{SABR and Immunotherapy}

In September of 2017, the PD-1 inhibitor nivolumab received accelerated FDA approval for advanced HCC after the phase I/II CheckMate-040 study showed an objective response rate of $15 \%$ (seven patients) including complete response in three, with disease control rate of $58 \%$, and a median response duration of 15 months [56••]. More recently, the results from the KEYNOTE-224 study, a phase II trial for pembrolizumab in patients with advanced $\mathrm{HCC}$, showed an objective response in $18(17 \%)$ of 104 patients, with one (1\%) complete and 17 (16\%) partial responses, and stable disease in 46 (44\%) patients [57•].

Radiation has been shown to favorably modulate the tumor immune microenvironment, cause a global change in immune stimulatory cytokine and chemokine profiles, promote a qualitative alteration in the $\mathrm{T}$ cell receptor repertoire, and enhance the tumoricidal phenotype of effector T cells [58・, 59•, 60, 61]. Synergistic effects of combined radiotherapy and immunotherapy, including checkpoint inhibitors, on local and distant tumor control have been described in various malignancies [58 $]$. The study of interaction between radiation and immunomodulating treatments is an area of active basic and translational cancer research. 
Tumors can evade immune responses. Evidence in support of this in $\mathrm{HCC}$ has been recently demonstrated in resected human HCC where the immune suppressive checkpoint receptors PD-L1 and LAG-3 (Lymphocyte-activation gene 3) were found to be upregulated with a concomitant decrease in density of intratumoral CD8 T cells [62]. Recently, Friedman et al. and Kim et al. showed improved therapeutic efficacy when combining RT with a checkpoint inhibitor in murine models of HCC [63, 64].

If synergy between SABR (or radiation in general) and immune therapy is seen in HCC, this could lead to a paradigm shift in HCC treatment. Not only would improvement in locoregional control in primary treatment setting be possible through radiosensitising immunotherapy, but improved therapeutic efficacy of immunotherapy in metastatic setting also could be achieved via abscopal effect with SABR. To this end, there is currently an ongoing phase 1 trial for SABR with nivolumab and ipilimumab [65].

\section{Conclusion}

SABR has emerged as an effective and safe non-invasive local therapy option for HCC in the primary and salvage treatment setting, as well as a bridge to liver transplantation in selected patients. SABR has demonstrated a high rate of tumor control with differing side effect profile relative to other local therapies. Active work in better predicting normal tissue toxicity and tumor radiosensitivity to guide tailored maximal safe treatment is under way and potential synergy with immune checkpoint inhibition and possibly other immunotherapies is also an ongoing area of research.

\section{Compliance with Ethical Standards}

Conflict of Interest Jeffrey Meyer reports clinical trial support from Peregrine Pharmaceuticals, Inc. and DFINE, Inc. And he also reports royalties from UpToDate, Inc. Byung-Han Rhieu and Amol K. Narang each declare no potential conflicts of interest.

Human and Animal Rights and Informed Consent This article does not contain any studies with human or animal subjects performed by any of the authors.

Open Access This article is distributed under the terms of the Creative Commons Attribution 4.0 International License (http:// creativecommons.org/licenses/by/4.0/), which permits use, duplication, adaptation, distribution, and reproduction in any medium or format, as long as you give appropriate credit to the original author(s) and the source, provide a link to the Creative Commons license and indicate if changes were made.

\section{References}

Papers of particular interest, published recently, have been highlighted as:
- Of importance

•- Of major importance

1. World Health Organization, International Agency for Research on Cancer. Globocan 2012: Estimated Cancer Incidence, Mortality and Prevalence Worldwide in 2012. http://globocan.iarc.fr/old/ FactSheets/cancers/liver-new.asp. Accessed 2 July 2018.

2. Llovet JM, Fuster J, Bruix J. Intention-to treat analysis of surgical treatment for early hepatocellular carcinoma: resection versus transplantation. Hepatology. 1999;30:1434-40.

3. Lo CM, Fan ST, Liu CL, Chan SC, Wong J. The role and limitation of living donor liver transplantation for hepatocellular carcinoma. Liver Transpl. 2004;10:440-7.

4. Mazzaferro V, Regalia E, Doci R, Andreola S, Pulvirenti A, Bozzetti F, et al. Liver transplantation for the treatment of small hepatocellular carcinomas in patients with cirrhosis. N Engl J Med. 1996;334:693-9.

5. Llovet JM, Ricci S, Mazzaferro V, Hilgard P, Gane E, Blanc JF, et al. Sorafenib in advanced hepatocellular carcinoma. N Engl J Med. 2008;359(4):378-90.

6. Bruix J, Qin S, Merle P, Granito A, Huang YH, Bodoky G, et al. Regorafenib for patients with hepatocellular carcinoma who progressed on sorafenib treatment (RESORCE): a randomised, double-blind, placebo-controlled, phase 3 trial. Lancet. 2017;389(10064):56-66.

7. Minami Y, Kudo M. Radiofrequency ablation of hepatocellular carcinoma: a literature review. Int J Hepatol. 2011;2011:104685.

8. Bruix J, Sherman M. Management of hepatocellular carcinoma. Hepatology. 2005;42(5):1208-36.

9. Sala M, Llovet JM, Vilana R, Bianchi L, Solé M, Ayuso C, et al. Initial response to percutaneous ablation predicts survival in patients with hepatocellular carcinoma. Hepatology. 2004;40(6): 1352-60.

10. Takaki H, Yamakado K, Nakatsuka A, Fuke H, Murata K, Shiraki $\mathrm{K}$, et al. Radiofrequency ablation combined with chemoembolization for the treatment of hepatocellular carcinomas measuring $5 \mathrm{~cm}$ or smaller: risk factors for local tumor progression. J Vasc Interv Radiol. 2007;18:856-61.

11. Fujimori M, Takaki H, Nakatsuka A, Uraki J, Yamanaka T, Hasegawa T, et al. Survival with up to 10 -year follow-up after combination therapy of chemoembolization and radiofrequency ablation for the treatment of hepatocellular carcinoma: single-center experience. J Vasc Interv Radiol. 2013;24:655-66.

12. Tateishi R, Shiina S, Teratani T, Obi S, Sato S, Koike Y, et al. Percutaneous radiofrequency ablation for hepatocellular carcinoma. An analysis of 1000 cases. Cancer. 2005;103:1201-9.

13. Nakazawa, Kokubu S, Shibuya A, Ono K, Watanabe M, Hidaka H, et al. Radiofrequency ablation of hepatocellular carcinoma: correlation between local tumor progression after ablation and ablative margin. AJR Am J Roentgenol. 2007;188:480-8.

14. Komorizono Y, Oketani M, Sako K, Yamasaki N, Shibatou T, Maeda M, et al. Risk factors for local recurrence of small hepatocellular carcinoma tumors after a single session, single application of percutaneous radiofrequency ablation. Cancer. 2003;97:125362.

15. Lencioni R, Cioni D, Crocetti L, Franchini C, Pina CD, Lera J, et al. Early-stage hepatocellular carcinoma in patients with cirrhosis: long-term results of percutaneous image-guided radiofrequency ablation. Radiology. 2005;234:961-7.

16. Llovet JM, Real MI, Montana X, et al. Arterial embolisation or chemoembolisation versus symptomatic treatment in patients with unresectable hepatocellular carcinoma: a randomised controlled trial. Lancet. 2002;359(9319):1734-9.

17. Llovet JM, Real MI, Montana X, et al. Arterial embolisation or chemoembolisation versus symptomatic treatment in patients with 
unresectable hepatocellular carcinoma: a randomised controlled trial. Lancet. 2002;359(9319):1734-9.

18. Molinari M, Kachura JR, Dixon E, et al. Transarterial chemoembolisation for advanced hepatocellular carcinoma: results from a North American cancer centre. Clin Oncol (R Coll Radiol). 2006;18(9): 684-92.

19. El-Serag HB, Marrero JA, Rudolph L, Reddy KR. Diagnosis and treatment of hepatocellular carcinoma. Gastroenterology. 2008;134(6):1752-63.

20. Shin SW, et al. The current practice of transarterial chemoembolization for the treatment of hepatocellular carcinoma. Korean $\mathrm{J}$ Radiol. 2009;10(5):425-34.

21. Lawrence TS, Robertson JM, Anscher MS, Jirtle R, Ensminger W, Fajardo L. Hepatic toxicity resulting from cancer treatment. Int $\mathrm{J}$ Radiat Oncol Biol Phys. 1995;31:1237-48.

22. Dawson LA, Normolle D, Balter JM, McGinn C, Lawrence T, Ten Haken R. Analysis of radiation induced liver disease using the Lyman NTCP model. Int J Radiat Oncol Biol Phys. 2002;53:810 21.

23. Reed GB Jr, Cox AJ Jr. The human liver after radiation injury. a form of veno-occlusive disease. Am J Pathol. 1966;48:597-611.

24. Timmerman RD, et al. Emergence of stereotactic body radiation therapy and its impact on current and future clinical practice. J Clin Oncol. 2014;32(26):2847-54.

25. Cárdenes HR, Price TR, Perkins SM, Maluccio M, Kwo P, Breen TE, et al. Phase I feasibility trial of stereotactic body radiation therapy for primary hepatocellular carcinoma. Clin Transl Oncol. 2010;12:218-25.

26. Andolino DL, Johnson CS, Maluccio M, Kwo P, Tector AJ, Zook J, et al. Stereotactic body radiotherapy for primary hepatocellular carcinoma. Int J Radiat Oncol Biol Phys. 2011;81:e447-53.

27. Kang J-K, Kim M-S, Cho CK, Yang KM, Yoo HJ, Kim JH, et al. Stereotactic body radiation therapy for inoperable hepatocellular carcinoma as a local salvage treatment after incomplete transarterial chemoembolization. Cancer. 2012;118:5424-31.

28. Bujold A, Massey CA, Kim JJ, Brierley J, Cho C, Wong RKS, et al. Sequential phase I and II trials of stereotactic body radiotherapy for locally advanced hepatocellular carcinoma. J Clin Oncol Off J Am Soc Clin Oncol. 2013;31:1631-9.

29. Lasley FD, Mannina EM, Johnson CS, Perkins SM, Althouse S, Maluccio M, et al. Treatment variables related to liver toxicity in patients with hepatocellular carcinoma, Child-Pugh class A and B enrolled in a phase 1-2 trial of stereotactic body radiation therapy. Pract Radiat Oncol. 2015;5:e443-9.

30. Takeda A, Sanuki N, Tsurugai Y, Iwabuchi S, Matsunaga K, Ebinuma $\mathrm{H}$, et al. Phase 2 study of stereotactic body radiotherapy and optional transarterial chemoembolization for solitary hepatocellular carcinoma not amenable to resection and radiofrequency ablation. Cancer. 2016;122:2041-9 A recent prospective trial demonstrating favorable efficacy and toxicity of SABR for HCC.

31. Pan CC, et al. Radiation-associated liver injury. Int J Radiat Oncol Biol Phys. 2010;76(3 Suppl):S94-100.

32. Rusthoven KE, Kavanagh BD, Cardenes H, Stieber VW, Burri SH, Feigenberg SJ, Chidel MA, Pugh TJ, Franklin W, Kane M, Gaspar LE, Schefter TE. Multi-institutional phase I/II trial of stereotactic body radiation therapy for liver metastases. J Clin Oncol. 2009;27(10):1572-1578.

33. Doi $\mathrm{H}$, et al. Threshold doses and prediction of visually apparent liver dysfunction after stereotactic body radiation therapy in cirrhotic and normal livers using magnetic resonance imaging. J Radiat Res. 2016;57(3):294-300.

34. Price RG, Apisarnthanarax S, Schaub SK, Nyflot MJ, Chapman TR, Matesan M, Vesselle HJ, Bowen SR. Regional radiation dose-response modeling of functional liver in hepatocellular carci- noma patients with longitudinal sulfur colloid SPECT/CT: a proof of concept. Int J Radiat Oncol Biol Phys 2018;3016(18):3100831023.

35. Toya R, et al. Dose-function histogram evaluation using $99 \mathrm{mTc}-$ GSA SPECT/CT images for stereotactic body radiation therapy planning for hepatocellular carcinoma patients: a dosimetric parameter comparison. Anticancer Res. 2018;38(3):1511-6.

36. Bowen SR, et al. Measuring total liver function on sulfur colloid SPECT/CT for improved risk stratification and outcome prediction of hepatocellular carcinoma patients. EJNMMI Res. 2016;6(1):57.

37. El Naqa I, et al. Modeling of normal tissue complications using imaging and biomarkers after radiation therapy for hepatocellular carcinoma. Int J Radiat Oncol Biol Phys. 2018;100(2):335-43.

38. Wahl DR, Stenmark MH, Tao Y, Pollom EL, Caoili EM, Lawrence TS, et al. Outcomes after stereotactic body radiotherapy or radiofrequency ablation for hepatocellular carcinoma. J Clin Oncol. 2016;34:452-9 A recent series suggesting a superior role of SABR over RFA for $\mathrm{HCC}$ lesions $\geq \mathbf{2} \mathbf{~ c m}$.

39. Rajyaguru DJ, et al. Radiofrequency ablation versus stereotactic body radiotherapy for localized hepatocellular carcinoma in nonsurgically managed patients: analysis of the National Cancer Database. J Clin Oncol. 2018;36(6):600-8.

40. Olsen JR, et al. Cross-modality comparisons between radiofrequency ablation and stereotactic body radiotherapy for treatment of hepatocellular carcinoma: limitations of the National Cancer Database. J Clin Oncol. 2018;26:JCO2018782904.

41. Sapir E, et al. Stereotactic body radiation therapy as an alternative to transarterial chemoembolization for hepatocellular carcinoma. Int $\mathrm{J}$ Radiat Oncol Biol Phys. 2018;100(1):122-30.

42. Coletta $\mathrm{M}$, et al. Bridging patients with hepatocellular cancer waiting for liver transplant: all the patients are the same. Transl Gastroenterol Hepatol. 2017;2:78.

43. She $\mathrm{WH}$, et al. Bridging and downstaging therapy in patients suffering from hepatocellular carcinoma waiting on the list of liver transplantation. Transl Gastroenterol Hepatol. 2016;1:34.

44. Moore A, et al. Stereotactic body radiation therapy (SBRT) for definitive treatment and as a bridge to liver transplantation in early stage inoperable Hepatocellular carcinoma. Radiat Oncol. 2017;12(1):163.

45. Rubinstein MM, et al. Bridging therapy effectiveness in the treatment of hepatocellular carcinoma prior to orthotopic liver transplantation. J Gastrointest Oncol. 2017;8(6):1051-5.

46. Sapisochin G, et al. Stereotactic body radiotherapy vs. TACE or RFA as a bridge to transplant in patients with hepatocellular carcinoma. An intention-to-treat analysis. J Hepatol. 2017;67(1):92-9.

47. Mohamed M, Katz AW, Tejani MA, Sharma AK, Kashyap R, Noel MS, et al. Comparison of outcomes between SBRT, yttrium-90 radioembolization, transarterial chemoembolization, and radiofrequency ablation as bridge to transplant for hepatocellular carcinoma. Adv Radiat Oncol. 2015;1:35-42.

48. O'Connor JK, Trotter J, Davis GL, Dempster J, Klintmalm GB, Goldstein RM. Long-term outcomes of stereotactic body radiation therapy in the treatment of hepatocellular cancer as a bridge to transplantation. Liver Transpl. 2012;8:949-54.

49. Facciuto ME, Singh MK, Rochon C, Sharma J, Gimenez C, Katta $\mathrm{U}$, et al. Stereotactic body radiation therapy in hepatocellular carcinoma and cirrhosis: evaluation of radiological and pathological response. J Surg Oncol. 2012;105:692-8.

50. Nugent F, et al. A randomized phase II study of individualized stereotactic body radiation therapy (SBRT) versus transarterial chemoembolization (TACE) with DEBDOX beads as a bridge to transplant in hepatocellular carcinoma (HCC). J Clin Oncol. 2017;35(No.4_suppl):223. https://doi.org/10.1200/JCO.2017.35. 4_suppl.223 An interim report of a prospective randomized 
trial comparing SABR with TACE as a bridge therapy to liver transplant, showing favorable efficacy and toxicity outcome with SABR.

51. Zeng ZC, et al. Consensus on stereotactic body radiation therapy for small-sized hepatocellular carcinoma at the 7th Asia-Pacific Primary Liver Cancer Expert Meeting. Liver Cancer. 2017;6(4): 264-74.

52. National Comprehensive Cancer Network. Hepatocellulcar carcinoma (Version 2.2018) https://www.nccn.org/professionals/ physician_gls/pdf/hepatobiliary.pdf. Accessed 3 July 2018.

53. Hong TS, et al. Multi-institutional phase II study of high-dose hypofractionated proton beam therapy in patients with localized, unresectable hepatocellular carcinoma and intrahepatic cholangiocarcinoma. J Clin Oncol. 2016;34(5):460-8.

54. Bush DA, et al. Randomized clinical trial comparing proton beam radiation therapy with transarterial chemoembolization for hepatocellular carcinoma: results of an interim analysis. Int J Radiat Oncol Biol Phys. 2016;95(1):477-82.

55. Bruix J, Sherman M. Practice Guidelines Committee, American Association for the Study of Liver Diseases: Management of hepatocellular carcinoma. Hepatology. 2005;42(5):1208-36.

$56 . \bullet$ El-Khoueiry AB, et al. Nivolumab in patients with advanced hepatocellular carcinoma (CheckMate 040): an open-label, non-comparative, phase $1 / 2$ dose escalation and expansion trial. Lancet. 2017;389(10088):2492-502. https://doi.org/10.1016/S01406736(17)31046-2 The prospective trial that led to the first FDA approval of immune checkpoint inhibitor for HCC.

57. Zhu AX, Finn RS, Edeline J, Cattan S, Ogasawara S, Palmer D, et al. Pembrolizumab in patients with advanced hepatocellular carcinoma previously treated with sorafenib (KEYNOTE-224): a nonrandomized, open-label phase 2 trial. Lancet Oncol. 2018. The most recent prospective trial that demonstrated efficacy of immune checkpoint inhibitor for HCC.;19:940-52.
58. Sharabi AB, et al. Radiation and checkpoint blockade immunotherapy: radiosensitisation and potential mechanisms of synergy. Lancet Oncol. 2015;16(13):e498-509 A recent comprehensive review on immune mechanisms of radiotherapy.

59. Twyman-Saint Victor C, et al. Radiation and dual checkpoint blockade activate non-redundant immune mechanisms in cancer. Nature. 2015;520(7547):373-7 Describes resistance mechanisms by which malignant tumor renders radiotherapy and immunotherapy ineffective, and demonstrates how mechanistic synergy of a combinatory therapy with radiation and multi-agent immunotherapy could overcome this.

60. Sharabi AB, et al. Stereotactic radiation therapy augments antigenspecific PD-1-mediated antitumor immune responses via crosspresentation of tumor antigen. Cancer Immunol Res. 2015;3(4): 345-55.

61. Chew V, Lee YH, Pan L, Nasir NJM, Lim CJ, Chua C, Lai L, Hazirah SN, Lim TKH, Goh BKP, Chung A, Lo RHG, Ng D, Filarca RLF, Albani S, Chow PKH. Immune activation underlies a sustained clinical response to Yttrium-90 radioembolisation in hepatocellular carcinoma. Gut. 2018. https://doi.org/10.1136/ gutjnl-2017-315485.

62. Yarchoan M, et al. Characterization of the Immune Microenvironment in Hepatocellular Carcinoma. Clin Cancer Res. 2017;23(23): 7333-9.

63. Friedman D, et al. Programmed cell death-1 blockade enhances response to stereotactic radiation in an orthotopic murine model of hepatocellular carcinoma. Hepatol Res. 2017;47(7):702-14.

64. Kim KJ, et al. Radiation improves antitumor effect of immune checkpoint inhibitor in murine hepatocellular carcinoma model. Oncotarget. 2017;8(25):41242-55.

65. ClinicalTrials.gov [Internet] Stereotactic Body Radiotherapy (SBRT) Followed by Immunotherapy in Liver Cancer. Available from: https://clinicaltrials.gov/ct2/show/NCT03203304. 